\title{
Screening for neuroblastoma in the north of England
}

\author{
L Parker, A W Craft, G Dale, S Bell, M Cole, A C McGill, J A Seviour, J Smith
}

Abstract

Objective-To determine the feasibility of establishing a system of screening for neuroblastoma.

Design-Prospective study of mass screening in four clearly defined geographical areas.

Setting-Four health districts of the Northern region of England.

Subjects-20829 babies aged 6 months, $92 \%$ of target population.

Interventions-Collection of urine on filter paper for analysis of content of homovanillic and vanillylmandelic acid in relation to urinary creatinine concentrations.

Main outcome measures-Derivation of reference range. Identification of babies with homovanillic or vanillylmandelic acid $>3 \mathrm{SD}$ above the mean (positive cases). Investigation of positive cases for evidence of neuroblastoma.

Results-The upper limit of normal (3 SD above the mean) for vanillylmandelic acid was $15 \mu \mathrm{mol} / \mathrm{mmol}$ creatinine and for homovanillic acid $24 \mu \mathrm{mol} / \mathrm{mmol}$ creatinine. Of the 20829 babies screened, $2537(12 \cdot 2 \%)$ required a second sample to be taken because the first sample was inadequate. Of these, $527(2 \cdot 5 \%)$ provided a liquid urine specimen and $10(0.04 \%)$ had positive results for neuroblastoma. Two of them had neuroblastoma (true positives) and eight did not (false positives). A further three children from the cohort were subsequently found to have neuroblastoma; they had raised homovanillic acid or vanillylmandelic acid values, or both, but screened negative at 6 months.

Conclusions-Screening for neuroblastoma is possible in the health care system of the United Kingdom. Evaluation of the efficacy of screening in reducing the mortality from neuroblastoma requires a controlled trial.

\section{Introduction}

Neuroblastoma is one of the most common solid

Department of Child

Health, The Medical

School, Newcastle upon Tyne NE2 4HH

L Parker, lecturer

A W Craft, professor

$\mathrm{S}$ Bell, health visitor

$\mathrm{M}$ Cole, research associate

$\mathrm{J}$ Smith, research associate

Department of Clinical Biochemistry, Newcastle General Hospital, Newcastle upon Tyne NE4 6BE

G Dale, senior lecturer A C McGill, principal biochemist

J A Seviour, senior chief medical laboratory scientific officer

Correspondence to:

Dr Parker. tumours of childhood, affecting one child in 10250 up to the age of 15 years. ${ }^{1}$ In almost half the cases the cancer is disseminated at diagnosis and the prognosis is appalling: even with aggressive chemotherapy followed by autologous bone marrow transplantation the most optimistic survival rate is $25 \%{ }^{2}$ The prognosis for neuroblastoma depends on the patient's age and the stage of the disease at presentation. ${ }^{3}$ Children aged less than 1 year at diagnosis who have either stage 1 or 2 disease have considerably better chances of survival.

Neuroblastoma is unusual among childhood malignancies in producing raised urinary concentrations of the catecholamine metabolites homovanillic and vanillylmandelic acid in over $90 \%$ of cases. ${ }^{4}$ It therefore seems to be an ideal candidate for a population based screening programme: it is a serious disease; it is an important cause of death in childhood; detection at a younger age and earlier stage improves the chances of survival; and there is the potential for a simple, noninvasive screening test. In fact, Sawada et al in Kyoto City, Japan, have pioneered a screening programme since the early 1970 s.

Preliminary results showed that their screening methods could detect cases of neuroblastoma, that the cases detected seemed to have a high chance of survival, and that early treatment was cost effective compared with treating cases detected clinically. ${ }^{5}$ On the basis of these preliminary studies screening was introduced for all 6 month old babies in Japan in $1985 .^{6}$ However, the effectiveness of any screening programme can be satisfactorily assessed only in a controlled trial comparing outcome in well matched screened and unscreened populations. The early reports from Japan indicated the apparent effectiveness of the screening programme from the improved survival of the cases detected. ${ }^{7}$ Unfortunately, problems of interpretation arise as a result of length time and lead time bias. Length time bias is the increase in the observed survival of cases detected by screening as a consequence of the higher proportion of slow growing (good prognosis) tumours in this group. Lead time bias is the increase in the observed survival of cases detected by screening as a result of the increased time that a particular case is observed between diagnosis and death as a direct consequence of earlier detection. The screening programme will probably also detect cases of neuroblastoma that might otherwise have remained undetected and regressed spontaneously ("silent" neuroblastoma); the only way to evaluate such screening is by comparing death rates from neuroblastoma in screened and unscreened populations. We carried out a pilot study in the north of England to assess the feasibility of setting up a controlled evaluation of neuroblastoma screening.

\section{Methods}

POPULATION AND SAMPLE COLLECTION

In 1985 a pilot project was begun among 6 month old babies registered with one health centre in North Tyneside. The project was then extended to four health districts: North Tyneside, South Tyneside, Gateshead, and North Tees. The total number of births in 1989 in the four districts was 9442 . During the study the policy of each of the districts was for all babies around 6 months old to have a routine health and developmental check carried out either at a health clinic or in the home by health visitors. In the United Kingdom all babies are registered with a health visitor (public health nurse), whose role is to monitor the health and development of all preschool children both during regular home visits and at infant welfare clinics.

The health visitors in the four districts discussed the question of neuroblastoma screening with mothers at an appropriate opportunity before the child was 6 months of age. The research nature of the investigation was explained to them and reiterated in a letter from the clinical director (AWC) of the study. Parents were warned that a repeat sample of urine might be required, in most cases for technical reasons rather than because their baby might have neuroblastoma. In the initial phase of the study the parent was asked to retain a freshly wet nappy on the day of the test, the sample being collected by the health visitor either at the clinic or at the baby's home. The health visitor extracted urine from the nappy by inserting a sheet of absorbent paper $(13 \mathrm{~cm}$ by $9 \mathrm{~cm}$; Schleicher and 
Schuell No 903) into the nappy, folding it over, and compressing it. The wet paper was placed in a plastic bag, sealed, attached to a form with an adhesive strip, and posted to the analytical laboratory at Newcastle General Hospital. Later in the study all parents in one health district (Gateshead) were asked to collect and dry the filter paper specimens themselves. The health visitor left them with a sample collection kit and parents were asked to collect the filter paper specimen and leave it to dry overnight (away from direct heat and light) before posting it to the laboratory.

\section{ANALYTICAL METHODS}

The paper (if wet) was dried before preparation and analysis. A section $\left(27 \mathrm{~cm}^{2}\right)$ was cut from the dried paper and an extract prepared for analysis. The creatinine content of the urine extract was determined by a picrate initial rate method on a Technicon RA500 analyser.

The aqueous samples and standards were acidified, extracted, and prepared for assay by gas chromatography and flame ionisation detection in the early part of the study and by gas chromatography mass spectrometry for most of the project. Details of both methods have been published. ${ }^{89}$

The homovanillic acid and vanillylmandelic concentrations in the urine extract were expressed as $\mu \mathrm{mol} / \mathrm{mmol}$ creatinine.

A reference range was established during the early phase of the study and modified as more samples were analysed. A value $3 \mathrm{SD}$ or more above the mean homovanillic acid and vanillylmandelic acid to creatinine ratios was taken as being an abnormal result. This arbitrary cut off value was similar to the cut off points used by the Japanese, and we had insufficient data at the inception of the project to determine a more sophisticated end point. A repeat assay was carried out on the remainder of the paper specimen if the initial result showed a creatinine concentration between 50 and $100 \mu \mathrm{mol} / 1$ (either because the urine was dilute or because insufficient urine had been absorbed on to the paper) or if the homovanillic acid or vanillylmandelic acid ratio, or both, was between 2 SD and 3 SD above the mean.

If the second analysis confirmed the low creatinine concentration or that either or both of the acid ratios were higher than $3 \mathrm{SD}$ above the mean, the health visitor was informed and a second urine specimen on filter paper requested. A second paper specimen was requested without a second assay having been performed on the first sample if the initial results showed a creatinine concentration less than $50 \mu \mathrm{mol} / 1$ or either or both of the acid ratios more than $3 \mathrm{D}$ above the mean. A liquid specimen was obtained if the second sample was inadequate, had a low creatinine concentration, or had a raised ratio of either or both of the acids. If the homovanillic acid or vanillylmandelic acid ratio, or both, remained 3 SD or more above the mean the health visitor was asked to bring the mother and baby to hospital for further evaluation. This consisted of a history and physical examination followed by chest radiography and abdominal ultrasonography. If an abnormality was found appropriate action was taken. The parents of babies whose clinical evaluation failed to show any evidence of neuroblastoma were reassured, and arrangements were made to collect repeat liquid urine samples at monthly intervals for six months.

Approval for the study was obtained from the four district ethical committees.

\section{Results}

Between 1986 and December 1990 a total of 20829 babies were screened in the four health districts. Reference ranges were established during the early phase of the study. The upper limit of normal (mean and $3 \mathrm{SD}$ ) for homovanillic acid was $24 \mu \mathrm{mol} / \mathrm{mmol}$ creatinine and for vanillylmandelic acid $15 \mu \mathrm{mol} / \mathrm{mmol}$ creatinine. For $2537(12 \cdot 2 \%)$ babies a second urine sample on filter paper was required, and for $527(2.5 \%)$ of them a liquid urine sample was required. Ten babies $(0.04 \%)$ had raised concentrations of homovanillic acid or vanillylmandelic acid, or both, and were referred for clinical evaluation. Of these, two were found to have a neuroblastoma and received treatment appropriate to stage (true positive cases; table I). In the remaining eight no evidence could be found of neuroblastoma and they were followed at monthly intervals (false positive cases; table I). A further three children from the same cohort of 20829 babies had negative results on screening but later presented clinically with neuroblastoma (false negatives; table II).

Compliance-A detailed audit was undertaken in 1989 in the North Tyneside Health District to determine the proportion of the target population who were screened. This was achieved by cross checking the birth register for the district against the list of children screened. Ninety three per cent of the births for that year had been screened.

Reference ranges-The initial reference range established during the early phase of the study was re-evaluated using 10000 first samples in which creatinine concentration was greater than $100 \mu \mathrm{mol} / \mathrm{l}$. This showed that the homovanillic acid and vanillylmandelic acid concentrations even when divided by creatinine were still dependent on creatinine concentration. Centiles were drawn up to allow for this and the resultant upper limits of normal are shown in table III. For homovanillic acid there was a difference in the spread of values obtained for boys and girls so separate reference ranges were established for each sex. For

TABLE I-Details of true and false positive cases of neuroblastoma detected at screening

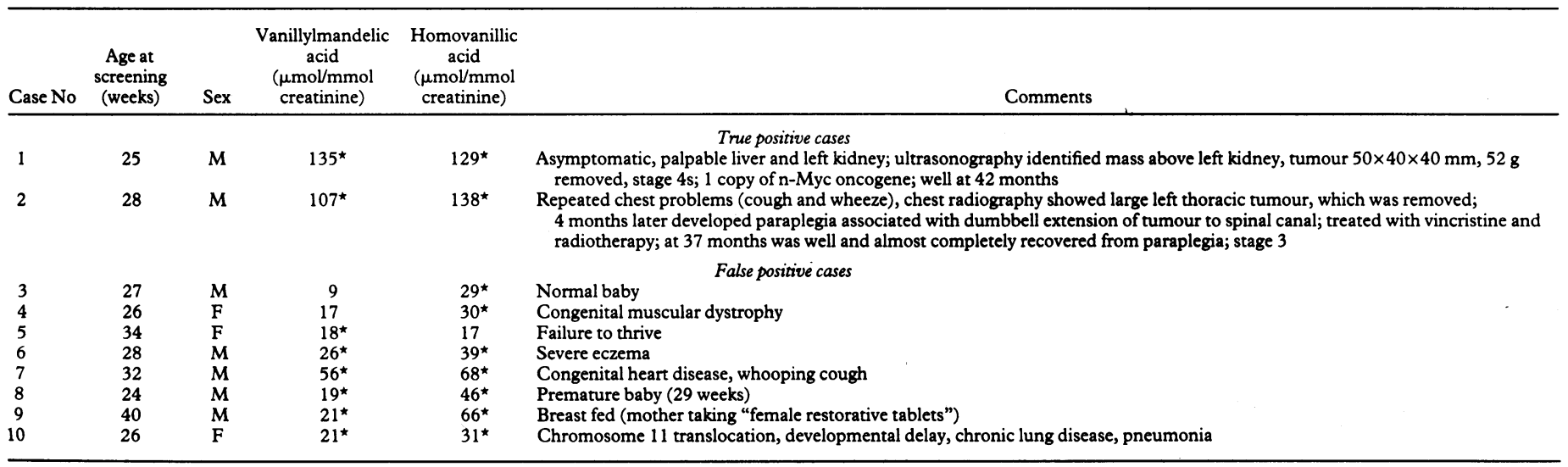

$\star$ Value $>3 \mathrm{SD}$ above mean. 


\begin{tabular}{|c|c|c|c|c|c|c|c|c|}
\hline Case No & $\begin{array}{l}\text { Age at } \\
\text { screening } \\
\text { (weeks) }\end{array}$ & Sex & $\begin{array}{c}\text { Vanillylmandelic } \\
\text { acid } \\
\text { ( } \mu \mathrm{mol} / \mathrm{mmol} \\
\text { creatinine })\end{array}$ & $\begin{array}{c}\text { Homovanillic } \\
\text { acid } \\
\text { ( } \mu \mathrm{mol} / \mathrm{mmol} \\
\text { creatinine) }\end{array}$ & $\begin{array}{l}\text { Age at } \\
\text { diagnosis } \\
\text { (months) }\end{array}$ & $\begin{array}{c}\text { Vanillylmandelic } \\
\text { acid } \\
\text { ( } \mu \mathrm{mol} / \mathrm{mmol} \\
\text { creatinine) }\end{array}$ & $\begin{array}{c}\text { Homovanillic } \\
\text { acid } \\
(\mu \mathrm{mol} / \mathrm{mmol} \\
\text { creatinine })\end{array}$ & Comments \\
\hline 11 & 26 & F & 16 & 12 & 28 & 39 & 46 & $\begin{array}{l}\text { Stage } 4 \text {, intensive chemotherapy, well } \\
\text { and disease free at } 48 \text { months }\end{array}$ \\
\hline 12 & 19 & F & 14 & 17 & 21 & $\uparrow$ & $\uparrow$ & $\begin{array}{l}\text { Stage } 4 \text {, intensive chemotherapy and } \\
\text { surgery, well at } 50 \text { months }\end{array}$ \\
\hline 13 & 26 & F & 6 & 6 & 33 & 34 & 40 & $\begin{array}{l}\text { Stage } 4 \text {, intensive chemotherapy and } \\
\text { surgery, died from recurrent tumour } \\
\text { at } 46 \text { months }\end{array}$ \\
\hline
\end{tabular}

TABLE III-Upper limit of normal values* for homovanillic and vanillylmandelic acids ( $\mu \mathrm{mol} / \mathrm{mmol}$ creatinine) in 6 month old infants

\begin{tabular}{|c|c|c|c|}
\hline \multirow[b]{2}{*}{ Creatinine $(\mu \mathrm{mol} / \mathrm{)})$} & \multicolumn{2}{|c|}{ Homovanillic acid } & \multirow{2}{*}{$\begin{array}{l}\text { Vanillyimandelic } \\
\text { acid }\end{array}$} \\
\hline & Boys & Girls & \\
\hline 100 & $104 \cdot 1$ & $45 \cdot 6$ & 35.5 \\
\hline 150 & 70.5 & $41 \cdot 4$ & $30 \cdot 0$ \\
\hline 200 & $55 \cdot 1$ & 38.4 & $26 \cdot 6$ \\
\hline 250 & $46 \cdot 1$ & $36 \cdot 1$ & $24 \cdot 0$ \\
\hline 300 & $40 \cdot 4$ & $34 \cdot 3$ & $22 \cdot 1$ \\
\hline 350 & $36 \cdot 3$ & $32 \cdot 7$ & $20 \cdot 6$ \\
\hline 400 & $33 \cdot 3$ & $31 \cdot 4$ & $19 \cdot 3$ \\
\hline 450 & $31 \cdot 1$ & $30 \cdot 2$ & $18 \cdot 2$ \\
\hline 500 & $29 \cdot 2$ & $29 \cdot 2$ & $17 \cdot 3$ \\
\hline 550 & $27 \cdot 7$ & 28.3 & 16.5 \\
\hline 600 & $26 \cdot 5$ & 27.5 & 15.8 \\
\hline 650 & $25 \cdot 5$ & $26 \cdot 7$ & $15 \cdot 2$ \\
\hline 700 & $24 \cdot 5$ & $26 \cdot 1$ & 14.6 \\
\hline 750 & 23.8 & 25.5 & $14 \cdot 1$ \\
\hline 800 & $23 \cdot 1$ & $24 \cdot 9$ & 13.6 \\
\hline 850 & 22.5 & $24 \cdot 3$ & 13.2 \\
\hline 900 & 22.0 & 23.8 & 12.8 \\
\hline 950 & 21.5 & $23 \cdot 3$ & 12.5 \\
\hline 1000 & $21 \cdot 1$ & 22.9 & $12 \cdot 1$ \\
\hline 1050 & $20 \cdot 7$ & 22.5 & 11.8 \\
\hline 1100 & $20 \cdot 4$ & $22 \cdot 1$ & 11.5 \\
\hline 1150 & $20 \cdot 1$ & 21.7 & $11 \cdot 2$ \\
\hline 1200 & 19.9 & $21 \cdot 4$ & 11.0 \\
\hline
\end{tabular}

ॠMean plus $3 \mathrm{SD}$ for normally distributed data.

vanillylmandelic acid there was no such sex difference. This analysis is discussed in more detail elsewhere. ${ }^{10}$

\section{Discussion}

This pilot study has confirmed previous reports from Japan that screening for neuroblastoma in 6 month old babies by measuring catecholamine metabolites in the urine is possible and can identify cases of neuroblastoma. ${ }^{5}$ The system is acceptable to both health visitors and parents, and there is a high compliance rate of $93 \%$ in the target population.

The high rate of requiring repeat urine samples is a cause for concern and was for two main reasons. Firstly, inadequate sample collection, with insufficient urine on the filter paper. As health visitors became more familiar with what constituted an adequate sample this problem diminished. Secondly, collection of excessively dilute urine with a creatinine content of less than $100 \mu \mathrm{mol} / \mathrm{h}$. If a baby was producing dilute urine a second urine sample was collected from an overnight nappy. However, creatinine may also have been low because of the practice of sending the filter papers to the laboratory without having dried them. This problem was recognised in the Japanese studies ${ }^{11}$ and results from infection of the wet filter paper by bacteria-notably, corynebacteria-which preferentially consume the creatinine, giving a spuriously low creatinine measurement and falsely raised homovanillic acid and vanillylmandelic acid ratios. In Japan this problem was overcome by drying the filter paper before it was sent to the laboratory. Since this practice was instituted during the later phase of our study the repeat rate has dropped substantially. A further, emerging, problem is the difficulty in obtaining sufficient urine from the new generation of ultra-absorbent nappies, and steps are being taken to overcome this problem. The high repeat rate did not seem to engender undue distress in parents, probably because they had been warned that a repeat request was likely to be for technical reasons. A detailed survey of health visitors' and parents' reactions to the study is underway.

\section{CASES DETECTED}

The two true positive cases identified both had a good prognosis according to category and stage of neuroblastoma, and both patients were successfully and simply treated. The three false negative cases all had a bad prognosis according to category of neuroblastoma, and one patient has already died. The two others showed no evidence of disease after intensive treatment. One of them (case 12) was screened early at 4 months as the family were leaving the area. Her homovanillic acid and vanillylmandelic acid were at the upper limit of normal and it is tempting to speculate that had she been screened two months later her neuroblastoma might have been identified. The eight false positive cases are worthy of some comment. In case 4 the low urinary concentrations of creatinine were probably the result of muscular dystrophy, giving a spuriously high vanillylmandelic acid to creatinine ratio. In four other cases $(5,6,7$, and 10$)$ the babies had serious chronic disease and the high concentrations of homovanillic acid and vanillylmandelic acid may merely indicate chronic stress with consequent raised catecholamines. ${ }^{12}$ All of these cases were designated as positive on the basis of the reference range, which was established at the beginning of the study. Reexamination of the results with the new centiles based on 10000 samples showed that five of the eight false positive cases would not have been designated positive, reducing the false positive rate to $0.01 \%$. This dependence of the ratios of homovanillic acid and vanillylmandelic acid to creatinine on the creatinine concentration of the urine has also been found by Tuchman et al, but they found no sex difference. ${ }^{13}$

\section{VALUE OF SCREENING}

Our study is too small to make any definitive statement about the value of neuroblastoma screening. The only way to evaluate efficacy is by studying the death rate from neuroblastoma in a defined population. The babies in this study were born in the Northern region, where the cumulative incidence of neuroblastoma from 1968 to 1985 was $1: 10850$ live births to age 15 years. In a cohort of 20829 babies two would be expected to have neuroblastoma before the age of 15 years. In this group five have already been found, considerably more than would be expected. The probability of diagnosing five or more cases based on local prescreening incidence rates is 0.0457 . Screening for neuroblastoma at 6 months of age may increase the incidence of the disease $\mathrm{e}^{14}$ by identifying cases that might otherwise have regressed spontaneously and therefore remained undetected. Only case 1 in the study could have come into this category. The probability of observing four or more cases in this population is $0 \cdot 128$. Neuroblastoma may be a heterogeneous disease, the tumours displaying either good or bad biological prognostic features-for example, nMyc 
amplification, ploidy, raised serum activities of ferritin and neurone specific enolase. ${ }^{15}$ Whether there is a progression from good to bad features remains controversial, although most of the evidence suggests that these features are constant within a given tumour. ${ }^{16}$

\section{EFFECTIVENESS AND FEASIBILITY OF SCREENING}

This study was set up to assess the feasibility of screening for neuroblastoma. Clearly this is possible and a high compliance rate can be achieved by using health visitors. By drying filter paper a low rate of taking repeat samples should be possible and there is a low false positive rate.

The authorities in Japan were convinced of the efficacy of screening so that since 1985 all babies in Japan have been offered screening. ${ }^{6}$ However, there is as yet no convincing evidence of a fall in the death rate from neuroblastoma in Japan. ${ }^{17} 18$ Because screening may detect silent cases of neuroblastoma, which might otherwise have spontaneously regressed, the only way to evaluate screening is a controlled trial comparing large screened and contemporaneously unscreened populations. Such a study is underway in Quebec ${ }^{13}$ and another has been proposed for the United Kingdom. ${ }^{19}$ Screening at 6 months of age has missed cases of neuroblastoma in this study, in Japan, ${ }^{20}$ and in Quebec (W G Woods, personal communication). This, together with the undoubted increase in incidence of neuroblastoma in screened populations as a result of the identification of silent cases calls into question the age at which children should be screened or indeed whether they should be screened at all. The age related mortality characteristics of neuroblastoma suggest that screening at around 15-18 months of age may well reduce the incidence of both false negative cases and silent neuroblastomas but whether this would influence the course of fatal cases requires further investigation. A definitive study of neuroblastoma screening is essential and will probably require one million babies to be screened and a similar number of controls. The results of such studies are awaited with interest before neuroblastoma screening is implemented in many other countries.

We thank the North of England Children's Cancer Research Fund, the Neuroblastoma Society, and the special trustees of the Newcastle hospitals. This study would not have been possible without the willing support of the health visitors of all four districts.

1 Craft AW, Amineddine HA, Scott JES, Wagget J. The Northern Region Children's Malignant Disease Registry, 1968-1982: incidence and survival. Br 7 Cancer 1987;56:853-8.

2 Shafford EA, Rogers DW, Pritchard J. Advanced neuroblastoma: improved response rate using a multiagent regimen (OPEC). 7 Clin Oncol 1984;2: $742-7$

3 Evans AE, D'Angio GJ, Propert K, Anderson J, Hann HW. Prognostic factors in neuroblastoma. Cancer 1987;59:1853-9.

4 Tuchman M, Ramnaraine MLR, Woods WG, Krivit W. Three years experience with random urinary homovanillic acid and vanillylmandelic acid levels in the diagnosis of neuroblastoma. Pediatrics 1987;79:203-5.

5 Sawada T, Hirayama M, Nakata T, Takeda T, Takasugi N, Mori T, et al. Mass screening for neuroblastoma in infants in Japan. Interim report of a mass screening study group. Lance 1984;ii:271-3.

6 Sawada T, Matsumara T, Matsuda Y, Kawakatsu H. Neuroblastoma studies in Japan. In: Miller AB, Chamberlain J, Day NE, Hakama M, Prorok PC, in Japan. In: Miller AB, Chamberlain J, Day NE, Hakama M, Prorok PC, eds. Cance $337-52$.

7 Nishi M, Miyake H, Takeda T, Shimada M, Takasugi N, Sato Y, et al. Effects of mass screening of neuroblastoma in Sapporo City. Cancer 1987;60:433-5. 8 Dale G, McGill A, Seviour JA, Craft AW. Urinary excretion of HMMA and homovanillic acid in infants. Ann Clin Biochem 1988;25:233-6.

9 Seviour JA, McGill AC, Dale G, Craft AW. Method of measurement of urinary homovanillic acid and vanillylmandelic acid by gas chromatography mass spectrometry suitable for neuroblastoma screening. $\mathcal{f}$ Chromatogr 1988;432 273-7.

10 Cole M, Parker L, Craft AW, Bell S, Dale G, McGill AC, et al. Creatinine related reference ranges for urinary HVA and VMA at 6 months of age. Arch Dis Child (in press)

11 Mizuta M, Umisa $H$. Utilisation of wet paper urine specimens in mass screening for neuroblastoma-the problem of bacterial contamination. fapan fournal of Public Health 1988;35:179-83.

12 Ruthven CRJ, Sandler M. Neurogenic amines and secreting tumours. In: Brown SS, Mitchell F, Young DS, eds. Chemical diagnosis of disease. Brown SS, Mitchell F, Young DS, eds. Chemi

13 Tuchman M, Lemieux B, Auray-Blais C, Robison LL, Giguere R, McCann $\mathrm{MT}$, et al. Screening for neuroblastoma at 3 weeks of age; methods and preliminary results from the Quebec Neuroblastoma Screening Project. Pediatrics 1990;86:765-73.

14 Nishi M, Miyake H, Takeda T, Shimada M, Takasugi N, Sato Y, et al Incidence of neuroblastoma in Sapporo City. I Pediatr Surg 1990;25:545-6.

15 Look AT, Hayes FA, Shuster JJ, Douglass EC, Castleberry RP, Bowman LC, et al. Clinical relevance of tumor cell ploidy and $\mathrm{n}$-myc gene amplification in childhood neuroblastoma: a Pediatric Oncology Group Study. 7 Clin Oncol 1991;9:581-91.

16 Brodeur GM, Hayes FA, Green AA, Casper JT, Wasson J, Wallach S, et al. Consistent $\mathrm{N}$-myc copy number in simultaneous or consecutive neuroConsistent $\mathrm{N}$-myc copy number in simultaneous or consecutive neuro-
blastoma samples from sixty individual patients. Cancer Res 1987;47: 4248-53.

17 Hanawa Y, Sawada T, Tsunoda A. Decrease in childhood neuroblastoma death in Japan. Med Pediatr Oncol 1990;18:472-5.

18 Cole M, Parker L, Craft AW. Decrease in childhood neuroblastoma death in Japan. Med Pediatr Oncol 1992;20:84-5.

19 Parker L, Craft AW, Dale G. Screening for neuroblastoma. Background preliminary experience in the North of England and proposals for an evaluative study. In: Miller AB, Chamberlain J, Day NE, Hakama M, Prorok PC, eds. Cancer screening. Cambridge: Cambridge University Press, 1991:337-52.

20 Nishi M, Miyake H, Takeda T, Takasugi N, Sato Y, Hanai J, et al. Cases of neuroblastoma missed by the mass screening programs. Pediatr Res 1989;26:603-9.

(Accepted 9 September 1992)

\section{Are patient consent forms for research protocols easy to read?}

Royal Brompton National Heart and Lung Hospital, London SW3 6NP

Kim A Priestley, research fellow

Claire Campbell, business administrator

Christopher B Valentine, research fellow

David M Denison, chairman, ethics committee

Nigel P Buller, senior lecturer

Correspondence to:

Dr K A Priestley,

Department of Invasive

Cardiology, Royal

Brompton National Heart

and Lung Hospital, London

SW3 6NP.

BMF 1992;305:1263-4

\section{Kim A Priestley, Claire Campbell, Christopher B \\ Valentine, David M Denison, \\ Nigel P Buller}

Written informed consent is an integral part of any clinical research protocol. It is solicited with a written consent form after a brief oral explanation of the project. Informed consent demands that the written explanation of the research project is easy to read and understand. Reading difficulty can be measured with several scoring systems.' ${ }^{\prime}$ Using established measurements of readability, we compared the readability of consent forms with that of daily newspapers.

\section{Methods and results}

We examined the consent forms of 50 protocols for research on adults. These were all the approved protocols submitted to the ethics committee of the
Royal Brompton National Heart and Lung Hospitals between 22 July 1991 and 8 April 1992. The consent forms were analysed with Microsoft Word for Windows, a computer software package that provides statistics on readability. Several variables were counted: words, characters, paragraphs, sentences, sentences per paragraph, words per sentence, and characters per word. Readability was measured with the Gunning fog index, the Flesch-Kincaid index, and the Flesch reading ease. These indices are derived from the length of sentences and the number of syllables per word or per 100 words.

For comparison we analysed the editorial and comment sections of 10 national newspapers published on one particular day (Daily Express, Daily Mail, Daily Mirror, Daily Star, Daily Telegraph, Guardian, Independent, Sun, Times, and Today). The mean scores for the consent forms and the newspapers were then compared by Student's $t$ test for unpaired data. Comparisons were also made with a mean score calculated for the three newspapers with the widest readership (Sun, Daily Mirror, and Daily Mail). ${ }^{2}$

The consent forms and newspaper editorials varied widely in length. This was reflected in the numbers of paragraphs, sentences, words, and characters used 